\title{
MicroRNA 3' end nucleotide modification patterns and arm selection preference in liver tissues
}

\author{
Sung-Chou Li ${ }^{1}$, Kuo-Wang Tsai ${ }^{2}$, Hung-Wei Pan², Yung-Ming Jeng ${ }^{3}$, Meng-Ru Ho ${ }^{4}$, Wen-Hsiung Li ${ }^{1,4,5^{*}}$ \\ From 23rd International Conference on Genome Informatics (GIW 2012) \\ Tainan, Taiwan. 12-14 December 2012
}

\begin{abstract}
Background: The expression of microRNA (miRNA) genes undergoes several maturation steps. Recent studies brought new insights into the maturation process, but also raised debates on the maturation mechanism. To understand the mechanism better, we downloaded small RNA sequence reads from NCBI SRA and quantified the expression profiles of miRNAs in normal and tumor liver tissues.

Results: From these miRNA expression profiles, we studied several issues related to miRNA biogenesis. First of all, the $3^{\prime \prime}$ ends of mature miRNAs usually carried modified nucleotides, generated from nucleotide addition or RNA editing. We found that adenine accounted for more than $50 \%$ of all miRNA $3^{\prime}$ end modification events in all libraries. However, uracil dominated over adenine in several miRNA types. Moreover, the miRNA reads in the HBV-associated libraries have much lower rates of nucleotide modification. These results indicate that miRNA $3^{\prime}$ end modifications are miRNA specific and may differ between normal and tumor tissues. Secondly, according to the hydrogen-bonding theory, the expression ratio of $5 p$ arm to $3 p$ arm miRNAs, derived from the same pre-miRNA, should be constant over tissues. However, a comparison of the expression profiles of the $5 p$ arm and $3 p$ arm miRNAs showed that one arm is preferred in the normal liver tissue whereas the other is preferred in the tumor liver tissue. In other words, different liver tissues have their own preferences on selecting either arm to be mature miRNAs.
\end{abstract}

Conclusions: The results suggest that besides the traditional miRNA biogenesis theory, another mechanism may also participate in the miRNA biogenesis pathways.

\section{Background}

MicroRNA (miRNA) genes are small non-protein-coding genes. Their final functional products are single-strand RNAs of $\sim 22$ nucleotides. MiRNAs repress the expression of their target genes. It has been commonly observed that in the tissues of genetic disorders or tumors, the expression levels of many miRNA genes are significantly different from those in the normal tissues [1-7]. In our previous study, we observed miRNA 3' end modification and arm selection preference in one pair of normal and tumor gastric tissues [8]. To better understand these phenomena, we analyzed NGS data of 15 liver tissues in this study. We first characterized the expression profiles of mature miRNAs in liver tissues by analyzing small RNA sequence

\footnotetext{
* Correspondence: wli@uchicago.edu

${ }^{1}$ Genomics Research Center, Academia Sinica, Taipei, Taiwan

Full list of author information is available at the end of the article
}

reads downloaded from NCBI SRA (SRP002272) [9]. Then, we used the expression profiles to address the issues to be described below.

The messenger RNAs (mRNAs) of protein-coding genes are usually subject to modifications, including poly-adenylation, which prolongs the lives of mRNAs. Recently, similar sequence modifications at the 3' end of miRNAs were detected by small RNA cloning and sequencing [10]. Further studies confirmed that such modifications were prevalent among animal and plant miRNAs and were caused by either RNA editing or nucleotide addition rather than by sequencing errors $[11,12]$. In this study, using the miRNA expression profiles in liver tissues, we showed that different tissues and different miRNAs have their own preferred nucleotide modifications although adenine accounted for the majority of the modification events. Moreover, HBV (hepatitis

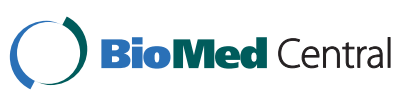

(c) 2012 Li et al.; licensee BioMed Central Ltd. This is an open access article distributed under the terms of the Creative Commons Attribution License (http://creativecommons.org/licenses/by/2.0), which permits unrestricted use, distribution, and reproduction in any medium, provided the original work is properly cited. 
B virus)-associated tissues exhibited lower adenine modification rates than normal liver tissues.

Although mature miRNAs are derived from pre-miRNAs, the expression of pre-miRNAs does not guarantee the expression of mature miRNA. In other words, not all premiRNAs are processed into mature miRNAs [13-15]. Moreover, the $5 \mathrm{p}$ arm and $3 \mathrm{p}$ arm of the same pre-miRNA usually have unequal likelihoods to be processed into mature miRNAs [16]. Such arm selection preference is commonly thought to result from the fact that the RISC unwinds the miRNA/miRNA* duplex at the end with weaker hydrogen binding. Therefore, the arm with the freer 5 ' end is preferentially incorporated into RISC to serve as the mature miRNA $[17,18]$. This hydrogen-bonding-based selection rule is currently the majority view.

However, recent studies showed that the orthologous pre-miRNAs, although highly similar to each other and thought to have the same miRNA/miRNA* duplex, showed preference of the $5 \mathrm{p}$ arm in one species but the $3 \mathrm{p}$ arm in another species. Therefore, the major and minor forms of mature miRNA in different species may be switched $[19,20]$. In this study, we are curious whether the same pre-miRNAs have different arm selection preferences between normal and tumor tissues. Using miRNA expression profiles, we showed that the major and minor mature miRNAs of the same pre-miRNA are not always consistent with the miRBase annotation. Moreover, we found that the $5 \mathrm{p}$ arm and $3 \mathrm{p}$ arm miRNA derived from the same pre-miRNA have different tissue expression preferences, one preferring the normal tissue while the other preferring the tumor tissue. The result points to the existence of another selection mechanism in addition to the hydrogen-bonding theory.

\section{Results and discussion}

\section{Analysis of sequence reads}

We downloaded small RNA sequence reads of 15 liver tissues for analysis. The accession number and description of each library are given in Table 1 . Under our criteria, there were about 8 to 11 million sequence reads available for analysis in each library except for SRX018968, which had only about 5.7 million reads (Table 1 ). Under our mapping criteria, about $80 \%$ to $90 \%$ of analyzed sequence reads were mapped to human miRNAs in most libraries. However, in SRX018968 and SRX018969, only about 64\% and $52 \%$ sequence reads, respectively, belonged to miRNAs. We were curious about the remaining reads and analyzed the non-miRNA reads in the libraries to see what kinds of molecules they were. We divided the reads into 10 categories (Additional File 1) and their frequencies are shown in Figure 1. The non-miRNA categories, especially mRNA $(\mathrm{p}<0.01)$ and tRNA $(\mathrm{p}<0.001)$, showed significantly higher percentages in SRX018968 and SRX018969 than in other libraries.
Vaz and colleagues observed a much lower miRNA percentage in small RNA collection from the K562 cell line, due to the reduced expression of the Dicer gene [21]. Among all of the 15 libraries we studied, only SRX018968 and SRX018969 were hepatitis C virus (HCV) associated libraries: SRX018969 was sampled from HCV-positive HCC tissue and SRX018968 was sampled from the adjacent region of $\mathrm{HCV}$-positive $\mathrm{HCC}$ tissue. Examining the miRNAs in these two libraries, we found that the first several abundant miRNAs are highly similar to the ones in the HCV-negative libraries. We wonder whether the infection of HCV represses the function of Drosha or Dicer so that the expressions of all miRNAs are evenly reduced, without altering the abundance ranking of individual miRNAs. Therefore, we examined the expression levels of Drosha and Dicer in HCV-positive and HCC-negative samples. Additional File 2 shows that the expression levels of Drosha and Dicer are not significantly different among samples. Thus, the lower numbers of miRNA reads in the two HCV-positive libraries could be simply due to sample preparation or the intrinsic characteristic of disease tissue.

In Morin's study, repeat elements accounted for about $16 \%$ of the total sequence reads from the human embryo stem cell [11]. In this study, however, repeat elements accounted for a very low percentage in all libraries. The difference might have occurred because we analyzed only the reads with 3' adapter ligated and excluded the one-copy clean reads.

\section{Analysis of detected miRNAs}

By mapping the sequence reads back to pre-miRNAs, one can quantify the expression levels of mature miRNAs. In this study, we detected about 500 to 600 mature miRNAs in most libraries. For each pre-miRNA, we arranged the miRNA reads according to the mapped locations within the pre-miRNA. As shown in Figure 2, hsa-mir-1307 encodes mature miRNA at only the $3 \mathrm{p}$ arm according to miRBase 16 . The integers denote the read counts. The numbers with comma in the right column denote the location offset relative to the reference miRNA annotated by miRBase. For example, the reads with " 0,0 " are exactly the same with the reference miRNAs. As reported previously $[11,22,23]$, we also observed that the reference miRNAs from miRBase are not necessary the most abundant ones. The mapping results of all pre-miRNAs in 15 libraries are shown in Additional File 3 (the read counts are not normalized as TPM, transcript per million).

Table 1 shows that about 500 to 600 mature miRNAs were detected in most libraries; however, most of the sequence reads belonged to a few miRNAs. For example, hsa-miR-122 accounted for $\geqq 50 \%$ of all reads in a library in most cases, except for SRX018961 (37.10\%) and SRX018971 (2.57\%). SRX018971 is the only HBV-negative and HCV-negative HCC sample. Hsa-miR-122 was 
Table 1 Summary of miRNA reads and library information.

\begin{tabular}{|c|c|c|c|c|c|c|c|}
\hline Library & $\begin{array}{l}\text { \# of reads } \\
\text { analyzed }\end{array}$ & $\begin{array}{l}\text { \# of miRNA } \\
\text { reads }\end{array}$ & $\begin{array}{l}\% \text { miRNA } \\
\text { reads }\end{array}$ & $\begin{array}{l}\text { \# of detected pre- } \\
\text { miRNAs }\end{array}$ & $\begin{array}{l}\text { \# of detected } \\
\text { miRNAs }\end{array}$ & $\begin{array}{l}\text { \# of detected miRNAs at } \\
\text { opposite arm }\end{array}$ & Description \\
\hline SRX018957 & $8,057,617$ & $7,025,801$ & $87.19 \%$ & 381 & 475 & 42 & Normal \\
\hline SRX018958 & $11,234,315$ & $10,241,350$ & $91.16 \%$ & 439 & 555 & 62 & Normal \\
\hline SRX018959 & $10,035,888$ & $9,229,254$ & $91.96 \%$ & 424 & 535 & 65 & Normal \\
\hline SRX018960 & $8,493,004$ & $7,424,150$ & $87.41 \%$ & 400 & 486 & 39 & $\mathrm{HBV}(+)$ \\
\hline SRX018961 & $8,214,755$ & $7,290,360$ & $88.75 \%$ & 507 & 609 & 82 & Severe HBV(+) \\
\hline SRX018962 & 7,413,793 & $5,767,890$ & $77.80 \%$ & 417 & 519 & 48 & HBV(+) distal \\
\hline SRX018963 & $8,214,714$ & $6,623,536$ & $80.63 \%$ & 418 & 529 & 51 & $\mathrm{HBV}(+)$ adjacent \\
\hline SRX018964 & $8,042,722$ & $6,668,203$ & $82.91 \%$ & 426 & 540 & 44 & $\mathrm{HBV}(+)$ side \\
\hline SRX018965 & $7,859,049$ & $6,332,172$ & $80.57 \%$ & 472 & 598 & 64 & $\mathrm{HBV}(+) \mathrm{HCC}$ \\
\hline SRX018966 & $10,640,841$ & $8,683,683$ & $81.61 \%$ & 467 & 596 & 60 & $\mathrm{HBV}(+)$ adjacent \\
\hline SRX018967 & $10,979,156$ & $9,790,644$ & $89.17 \%$ & 465 & 584 & 64 & $\mathrm{HBV}(+) \mathrm{HCC}$ \\
\hline SRX018968 & $5,686,232$ & $3,663,927$ & $64.44 \%$ & 443 & 556 & 58 & $\mathrm{HCV}(+)$ adjacent \\
\hline SRX018969 & $9,224,742$ & $4,754,658$ & $51.54 \%$ & 430 & 521 & 68 & $\mathrm{HCV}(+) \mathrm{HCC}$ \\
\hline SRX018970 & $8,967,260$ & $7,618,011$ & $84.95 \%$ & 407 & 519 & 56 & $\begin{array}{l}\text { HBV(-) HCV(-) } \\
\text { adjacent }\end{array}$ \\
\hline SRX018971 & $9,102,563$ & $7,259,865$ & $79.76 \%$ & 546 & 682 & 112 & $\begin{array}{l}\mathrm{HBV}(-) \mathrm{HCV}(-) \\
\mathrm{HCC}\end{array}$ \\
\hline
\end{tabular}

We mapped the sequence reads back to human pre-miRNAs, quantifying the expression levels of pre-miRNAs, mature miRNAs and isomiRs. According to miRBase 16, 730 out of the 1,048 human pre-miRNAs encode only one mature miRNA at one arm, either $5 p$ or $3 p$ arm. With the improvement on NGS's sequencing depth, we also detected the opposite-arm mature miRNAs at the pre-miRNAs originally annotated to encode only one miRNA. HBV, HCV and HCC are abbreviations of hepatitis $B$ virus, hepatitis $C$ virus and hepatocellular carcinoma, respectively. Distal, adjacent and side mean that the sites at which the tissues were sampled were $>10 \mathrm{~cm}, 2-5 \mathrm{~cm}$ and $0-2 \mathrm{~cm}$ far from the HCC sites.

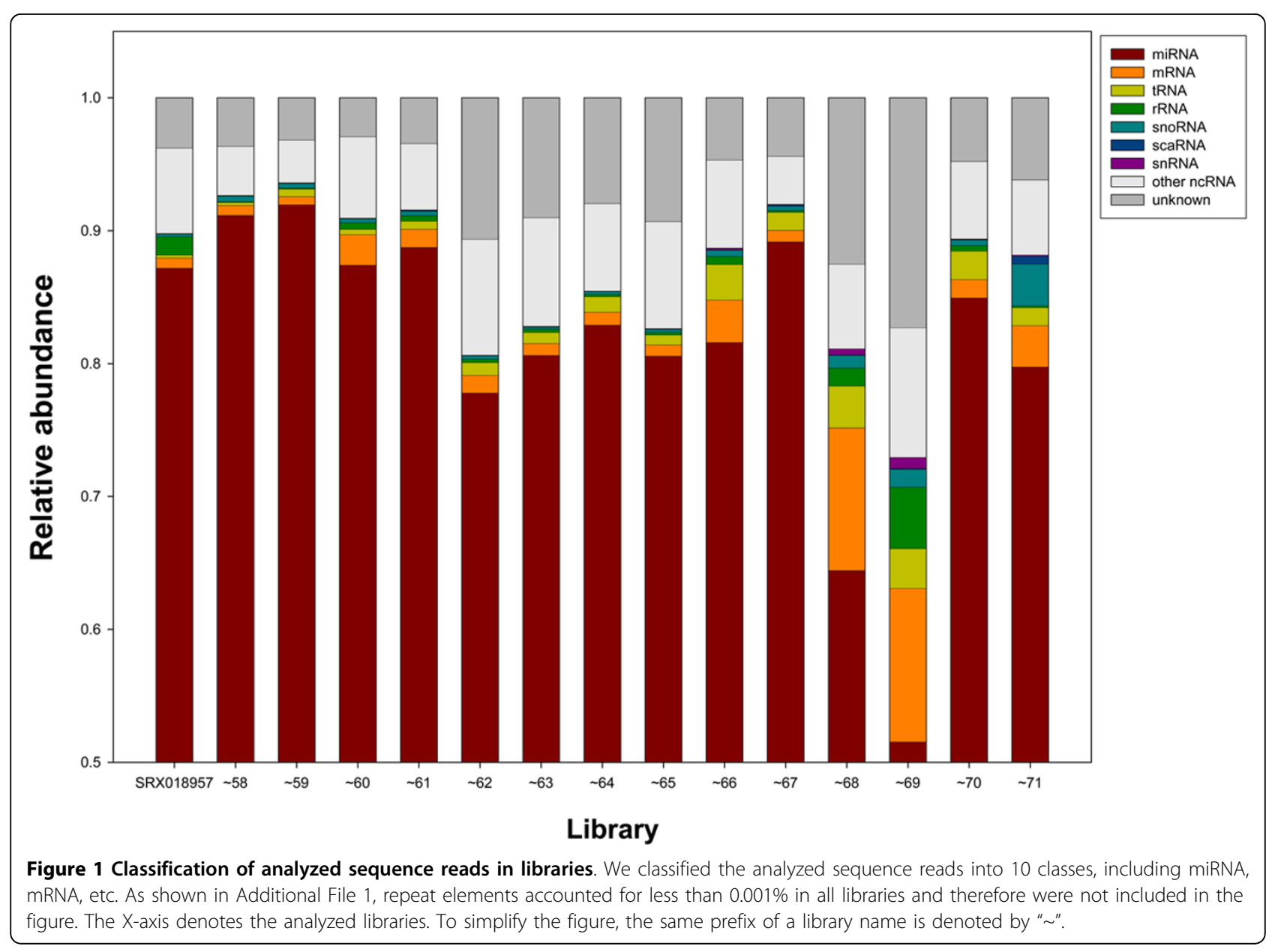




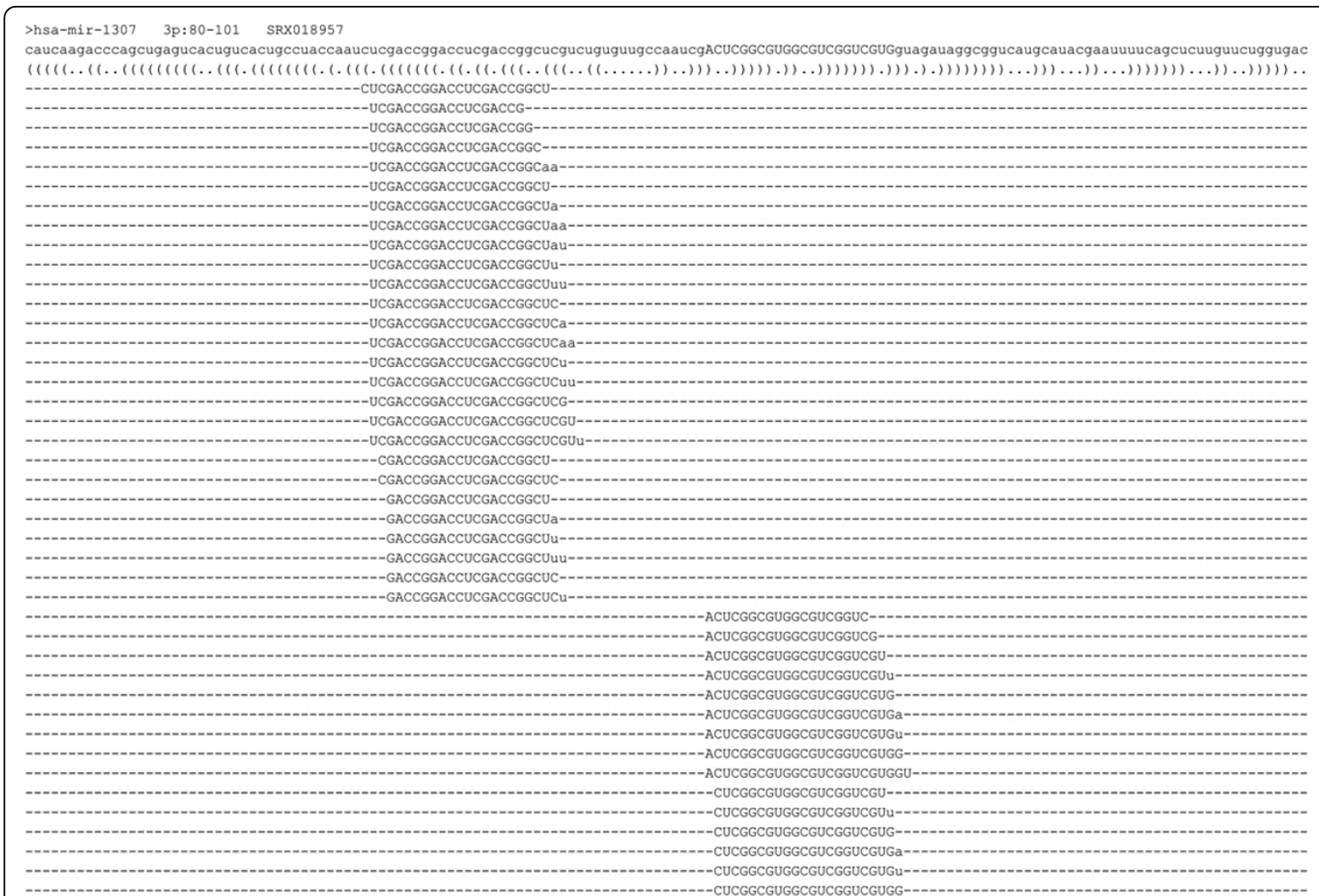

Figure 2 Mapping result of hsa-mir-1307 reads in the SRX018957 library. Hsa-mir-1307 encodes mature miRNA hsa-miR-1307 at its 3p arm ranging from nt 80 to 101 of pre-miRNA, as presented in uppercase. The sequence reads mapped to hsa-mir-1307 were arranged according to their mapped loci within the pre-miRNA. The expression level of a mature miRNA is determined by summing the read counts (initial read counts, not normalized as TPM, transcript per million) of all its iso-reads, isomiRs. The mismatches at the $3^{\prime}$ ends, shown in lowercase, denoted the modified fragments. In the figure, only the modified fragments, including $A, U, A A, A U$ and $U U$, with frequency $\geqq 1 \%$ were recovered.

reported to activate HCV translation [24] and to be highly expressed in HCV infected patients [25]. However, comparing with other HCV-positive (SRX018968 and SRX018969) and HCV-negative samples, the expression level of hsa-miR-122 in SRX018971 dropped dramatically. This is an interesting observation for further study.

\section{Detection of additional miRNAs at the opposite arms}

Among the 1,048 human pre-miRNAs, 730 were annotated to encode mature miRNAs at only one arm in miRBase 16. With the increased sequencing depth of NGS platforms, more mature miRNA can be detected at the opposite arm. As shown in Figure 2, we detected also the mature miRNA at the opposite arm, i.e., the $5 \mathrm{p}$ arm of hsa-mir-1307 from SRX018957. Furthermore, like common mature miRNAs, the opposite-arm miRNA of hsamir-1307 also has many isomiR types, highly overlapping with each other. Actually, in all of the 15 libraries, the opposite-arm miRNA of hsa-mir-1307 was detected.

As described in a previous study [22], the opposite-arm miRNAs are not necessarily kept at lower expression levels than the annotated ones. In this study, the opposite-arm
miRNA of hsa-mir-1307 has a higher expression level than the annotated one in most libraries, except for SRX018968 and SRX018971. In other pre-miRNAs, this phenomenon can also be observed (Additional File 3), which challenges the nomenclature of miRBase. The finding of oppositearm miRNAs was made because we mapped the sequence reads back to pre-miRNAs rather than to mature miRNAs. In this study, we detected many opposite-arm miRNAs in all 15 libraries (Table 1).

\section{Analysis of modification fragments generated by $3^{\prime}$ end modification}

Previous studies reported nucleotide variations preferentially locating at the 3' end of miRNAs [10-12,26,27]. Such variations can arise from either nucleotide additions, elongating the miRNAs, or RNA editing that does not alter the miRNA length. Regardless of how the modified nucleotides occur, they can cause mismatches in the mapping procedure, making the originally perfect match reads fail to be mapped back to miRNAs. Therefore, we trimmed the terminal 3' end mismatch one nucleotide at one time until mapping succeeded, and then, analyzed the trimmed 
nucleotides. These trimmed nucleotides are thought to have occurred by modifications so that we call them modified fragments, which were recovered and presented in lower case (Figure 2 and Additional File 3).

Table 2 shows that in most libraries the most abundant modified fragments are A, U, AA, AU and UU in the descending order of relative abundance, where $A$ and $U$ denoted adenine and uracile, respectively. These modified fragments are AU rich and A accounts for at least $50 \%$ of all modification events in all libraries. In addition, onenucleotide modifications (A and $\mathrm{U}$ ) are much more frequent than two-nucleotide modifications (AA, AU, UU, etc). In summary, these five modification events account for almost $90 \%$ of all modification events in most libraries except for SRX018965 and 71 (ie., SRX018971).

Moreover, almost half of all libraries, including SRX018957, 58, 59, 60, 66, 67 and 70, had about $30 \%$ of miRNAs reads undergone modification at their 3 ' ends. The modification rate ranged from $13 \%$ to $20 \%$ in the remaining libraries. Upon further examination, most of the low-modification-rate libraries belong to hepatitis B virus (HBV) associated samples (including SRX018961, $\sim 62, \sim 63, \sim 64$ and $\sim 65$ ). Furthermore, we found that the reduced modification rates were mainly owing to the decrease of A modification (Figure 3a), implying that HBV infection can repress the mechanisms responsible for the A modification without affecting the other modifications. In summary, with our mapping method, we can use these modified sequence reads to quantify miRNAs.

Figure 3a shows the global distribution patterns of modification events in libraries. However, we are more interested in whether individual miRNAs have preferred modifications different from the global pattern. Therefore, we examined 16 mature miRNAs with a high expression level in most libraries. We found that four mature miRNAs, miR-30d, miR-101, miR-140-3p and miR-378, have similar patterns to the global patterns (Figure 3a). However, in let-7a, let-7b, let-7c, miR-122 and miR148a, the patterns are somewhat different from the global pattern. For example, in miR-122 (Figure $3 \mathrm{~b}$ ), the frequencies of $U$ and UU events decreased so dramatically in all libraries that $U$ was even much rarer than AA, which was originally dominated by $\mathrm{U}$ in the global pattern.

Another interesting pattern can be illustrated by miR192 and miR-29a. Contrary to the pattern of miR-122, the frequencies of $\mathrm{A}$ and AA events dramatically decreased in miR-192 (Figure 3c); in other words, U and UU dominated over other modification events except in the SRX018971 library. The A and U decreases in Figure $3 \mathrm{c}$ and $\mathrm{3b}$ were consistently found in almost all libraries, demonstrating that different individual miRNAs may have their own preferred modifications.

Although the above modification patterns were consistent in almost all libraries, we observed inconsistent patterns in miR-99a, miR-100, miR-103, miR-191 and miR199a-3p. For example, in miR-199a-3p (Figure 3d), the U modification has a significantly higher frequency than the A modification in SRX018960, 61, 62, 63, 64 and $\sim 65$, most of which are HBV associated libraries. However, the A modification obviously dominated over the $U$ modification in the remaining libraries, most of which were not HBV associated libraries. The global pattern of modifications (Figure 3a) implied that HBV infection can repress the A modification, which seems to be consistent with the result for miR-199a-3p (Figure 3d).

Previous studies reported several types of RNA editing, such as A to $G$ transition catalyzed by adenine deaminase and $\mathrm{C}$ to $\mathrm{U}$ transition catalyzed by cytidine

Table 2 Frequencies of the $3^{\prime}$ end modified fragments in libraries.

\begin{tabular}{cllllllc}
\hline Library & A & U & AA & AU & UU & SUM & miRNA reads with 3' end modi. \\
\hline SRX018957 & $64.74 \%$ & $11.86 \%$ & $6.46 \%$ & $4.61 \%$ & $4.58 \%$ & $92.25 \%$ & $29.78 \%$ \\
SRX018958 & $67.17 \%$ & $11.95 \%$ & $4.98 \%$ & $2.77 \%$ & $3.90 \%$ & $90.77 \%$ & $30.68 \%$ \\
SRX018959 & $73.16 \%$ & $9.40 \%$ & $4.90 \%$ & $2.72 \%$ & $2.47 \%$ & $92.65 \%$ & $28.55 \%$ \\
SRX018960 & $72.54 \%$ & $11.39 \%$ & $5.06 \%$ & $2.29 \%$ & $2.56 \%$ & $93.84 \%$ & $30.11 \%$ \\
SRX018961 & $60.76 \%$ & $19.78 \%$ & $5.57 \%$ & $2.83 \%$ & $2.04 \%$ & $90.98 \%$ & $15.02 \%$ \\
SRX018962 & $66.21 \%$ & $13.10 \%$ & $5.31 \%$ & $3.89 \%$ & $2.72 \%$ & $91.23 \%$ & $19.90 \%$ \\
SRX018963 & $64.39 \%$ & $14.56 \%$ & $5.52 \%$ & $3.82 \%$ & $3.34 \%$ & $91.63 \%$ & $20.31 \%$ \\
SRX018964 & $60.02 \%$ & $16.08 \%$ & $5.96 \%$ & $4.81 \%$ & $4.37 \%$ & $91.24 \%$ & $20.55 \%$ \\
SRX018965 & $62.39 \%$ & $11.89 \%$ & $4.22 \%$ & $3.66 \%$ & $1.61 \%$ & $83.77 \%$ & $12.91 \%$ \\
SRX018966 & $68.71 \%$ & $10.60 \%$ & $5.92 \%$ & $1.17 \%$ & $2.94 \%$ & $89.34 \%$ & $27.19 \%$ \\
SRX018967 & $73.14 \%$ & $8.56 \%$ & $6.30 \%$ & $2.70 \%$ & $1.77 \%$ & $92.47 \%$ & $31.05 \%$ \\
SRX018968 & $69.95 \%$ & $8.60 \%$ & $7.66 \%$ & $3.17 \%$ & $1.66 \%$ & $91.04 \%$ & $21.93 \%$ \\
SRX018969 & $63.61 \%$ & $14.26 \%$ & $5.01 \%$ & $2.59 \%$ & $4.18 \%$ & $89.65 \%$ & $18.28 \%$ \\
SRX018970 & $67.59 \%$ & $9.56 \%$ & $4.80 \%$ & $3.99 \%$ & $4.82 \%$ & $90.76 \%$ & $35.91 \%$ \\
SRX018971 & $50.00 \%$ & $14.03 \%$ & $11.32 \%$ & $4.48 \%$ & $2.42 \%$ & $82.25 \%$ & $16.81 \%$ \\
\hline
\end{tabular}

Using an alternative mapping criterion, we collected the sequences of the $3^{\prime}$ end modified fragments by analyzing their relative abundances in all modification events. $A$ and $U$ account for almost $80 \%$ of all modification events. Only the modified fragments accounting for $\geqq 1 \%$ of all modification events are listed here. 
a

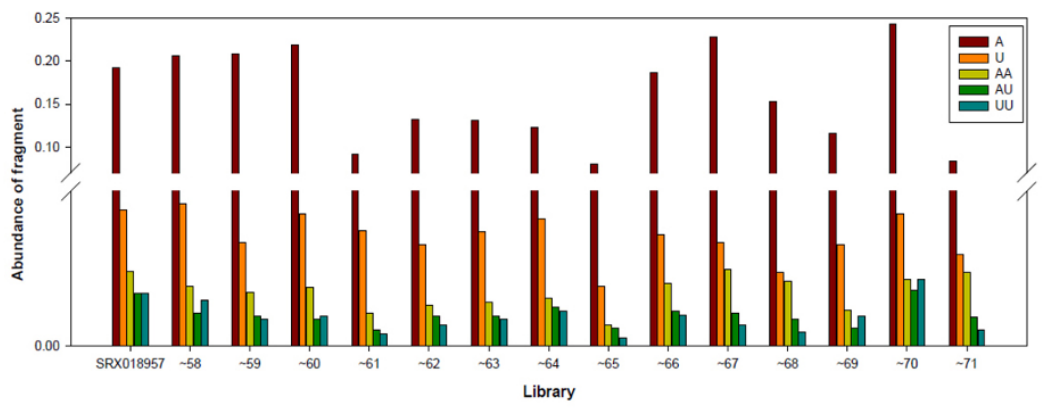

b

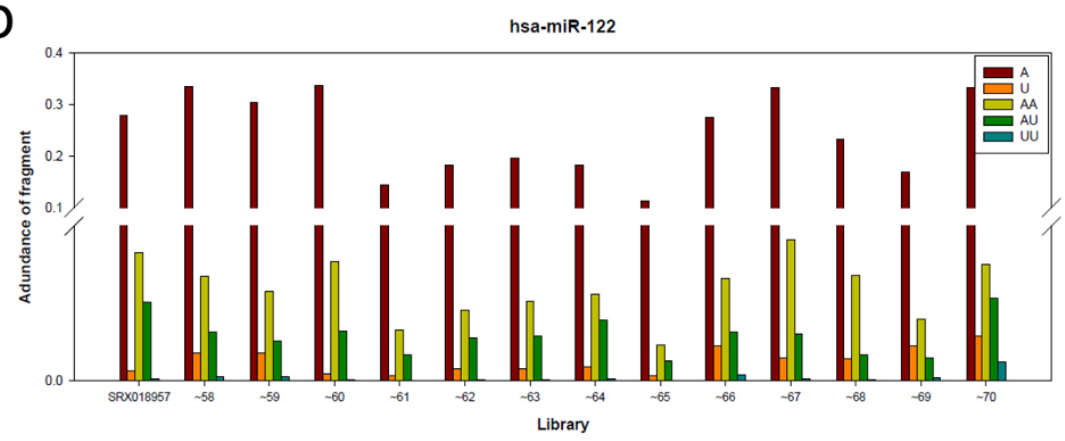

C

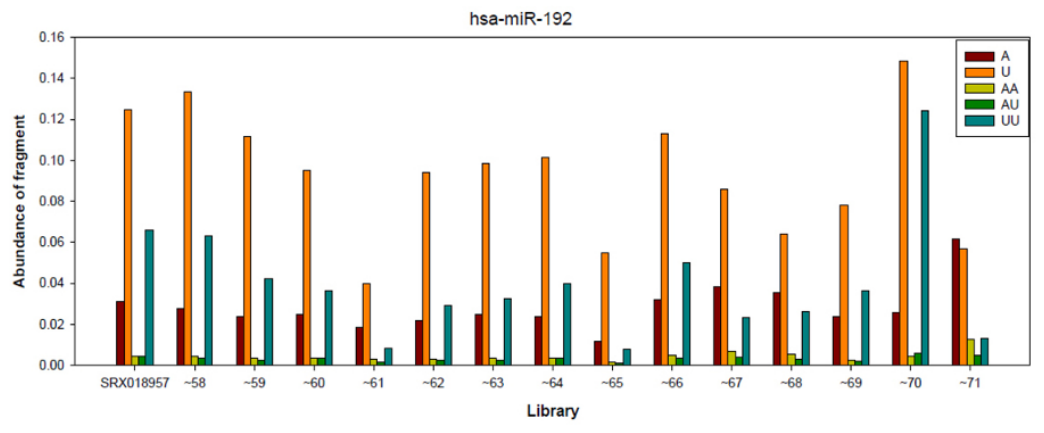

d

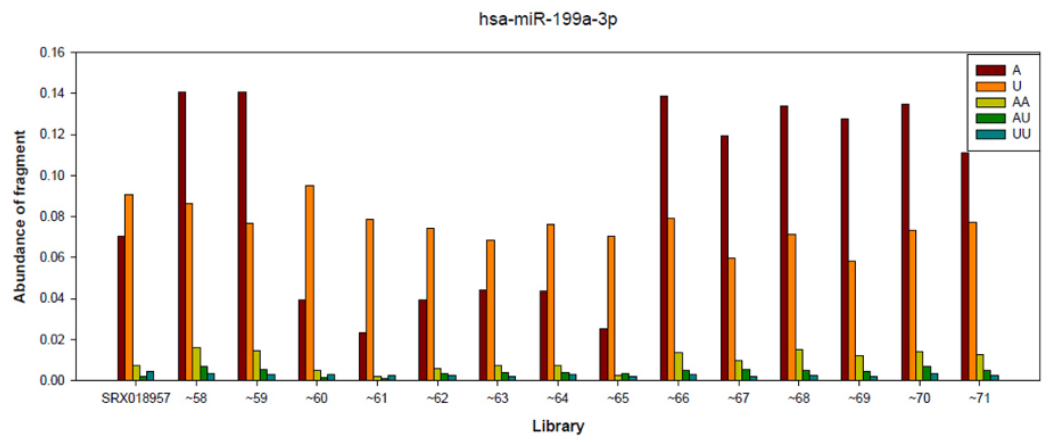

Figure 3 The frequencies of abundant modified fragments in all libraries. Only the modification events with $\geqq 1 \%$ frequency in all libraries were plotted. (a) The global patterns of modification events. (b,c) Several miRNAs have their specific patterns of modification events different from the global one. The expression level of hsa-miR-122 is much lower in SRX018971 than in other libraries, so that SRX018971 was not included in Figure 2a. (d) The patterns of modification events of some miRNAs are library specific. 
deaminase [11,28], responsible for generating 3' end variations without altering the miRNA length. However, nucleotide addition can also cause the same variations by elongating miRNA length. Owing to the existence of isomiR, the lengths of miRNAs can be dynamic [29]. Therefore, it is difficult to determine whether nucleotide addition or RNA editing contributes to such 3' end variations. Hence, instead of RNA editing or nucleotide addition, we used the term "modifications" to avoid the debate. In summary, 3' end modifications can be miRNA dependent and can depend on the tissue condition (normal or abnormal), making the modification patterns more complicated.

In this study, we showed that the 3' end modification event of miRNA cab be library dependent and that HBVpositive samples tend to have a lower chance of A modification. MiRNAs recognize their target site mainly by the complementary pairing between their seed region, nucleotides 2 7, and the 3' UTR. The modification event occurring in the 3' end can hardly alter their selection of target gene. Therefore, 3 ' end modification may have little impact on miRNAs' target gene selection and function.

\section{Inconsistent expression ratios of $5 p$ arm to $3 p$ arm}

According to the hydrogen-bonding theory, the selection preference between pre-miRNA's $5 p$ arm and 3p arm is an intrinsic characteristic of pre-miRNA [17]. If this selection mechanism is the only one in deciding arm selection preference, the expression ratio of $5 p$ arm to $3 p$ arm should be largely constant in all tissues. To examine this theory, we investigated whether for a pre-miRNA the expression ratio of $5 p$ arm to $3 p$ arm differs between normal and tumor tissues. As shown in Figure 2, the $5 p$ and $3 p$ sequence reads of hsa-mir-1307 in SRX018957 were, respectively, 1325 and 402, resulting in a 3.30 expression ratio of $5 \mathrm{p}$ arm to $3 \mathrm{p}$ arm.

Among the 15 libraries, SRX018957, 58 and 59 were three replicates of normal liver tissue, while SRX018965 and $\sim 67$ were two replicates of HBV-positive HCC (HBV $(+) \mathrm{HCC})$ tissue. We compared the expression ratios averaged from the three normal and the two $\mathrm{HBV}(+) \mathrm{HCC}$ liver tissues. In Figure 4a, an upward bar means that the $5 p$ arms had a larger read count than the $3 p$ arm, while a downward bar means that the 3p arm was the major arms in terms of abundance. Among the 49 examined pre-

a

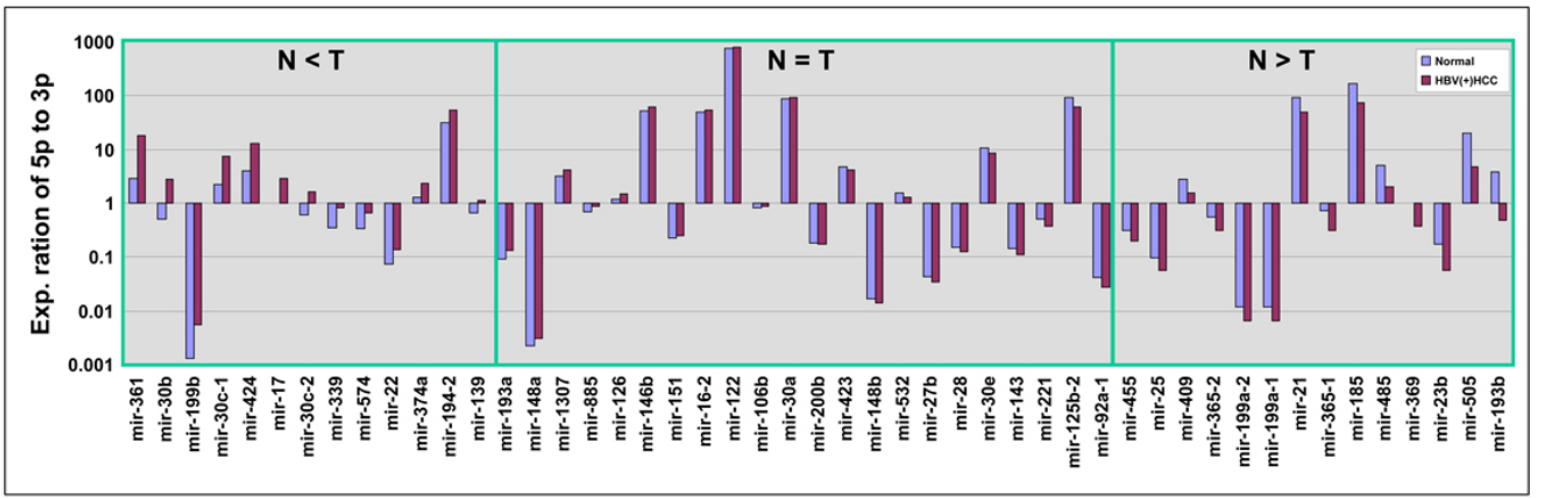

$\mathrm{b}$

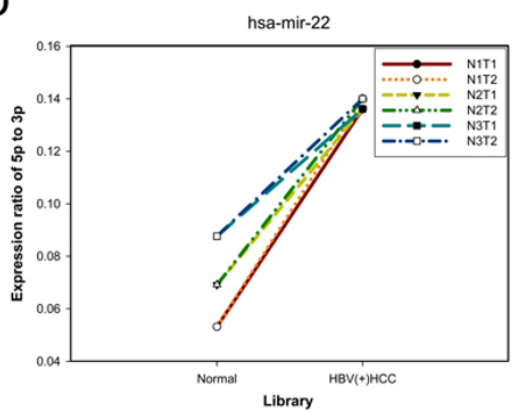

c

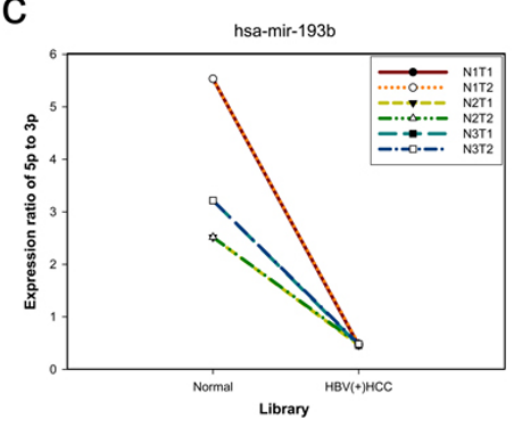

d

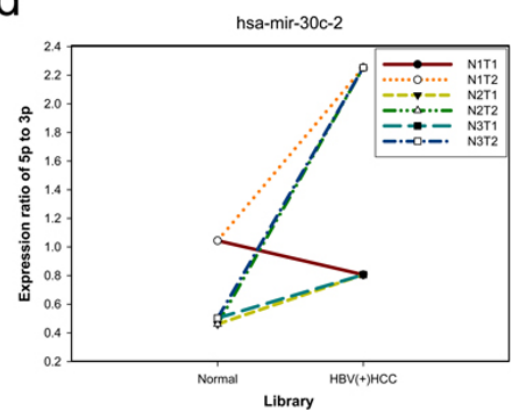

Figure 4 The expression ratios of $5 p$ arm miRNA to $3 p$ arm miRNA. In order to avoid extreme values caused by low expression levels, we examined only 49 pre-miRNAs whose read counts of $5 p$ arm and $3 p$ arm were $\geq 5$ in TPM (transcript per million) unit. The cutoff of significant difference of expression ratios between libraries is 1.5 fold change. (a) The expression ratios of $5 p$ arm to $3 p$ arm were averaged from three normal and two HBV(+)HCC libraries. (b,c,d) The N1, N2, N3, T1 and T2 denoted SRX018957, SRX018958, SRX018959, SRX018965 and SRX018967 libraries, respectively. So, NIT1 denoted the comparison in the NT pair composed of SRX018957 and SRX018965. 
miRNAs, 27 have unequal averaged expression ratios between normal and $\mathrm{HBV}(+) \mathrm{HCC}$ tissues under the cutoff of 1.5 fold change. Moreover, 13 and 14 out of the 27 premiRNAs had higher expression ratios in tumor and normal tissues, respectively. Figure 4a shows that more than half of the examined pre-miRNAs had unequal expression ratios and several of them had a fold change up to 10, demonstrating that in many pre-miRNAs the arm selection preference is different between the normal liver and $\mathrm{HBV}(+) \mathrm{HCC}$ tissues.

Although Figure 4a shows a significant difference, an analysis based on average values may neglect the diversities among tissues. Hence, we also examined pre-miRNAs' expression ratios of $5 p$ arm to $3 p$ arm among six normal HBV $(+) \mathrm{HCC}(\mathrm{NT})$ pairs. Several pre-miRNAs showed consistent patterns between NT pairs. Figure 4b shows that hsa-mir-22's expression ratios of $5 p$ arm to $3 p$ arm had the same tendency between NT pairs and that they were all higher in $\mathrm{HBV}(+) \mathrm{HCC}$ tissues than in normal tissues. This result reflected the fact that the $5 \mathrm{p}$ arm of hsa-mir-22 was more preferred in HBV(+)HCC tissues than in normal tissues. A similar phenomenon was also observed in other pre-miRNAs such as hsa-mir-17 and hsa-mir-30b.

Although hsa-mir-193b's expression ratios of $5 \mathrm{p}$ arm to $3 p$ arm also had the same tendency between NT pairs, the values were all higher in normal tissues rather than in $\mathrm{HBV}(+) \mathrm{HCC}$ tissues (Figure 4c). Furthermore, the 5p arm of hsa-mir-193b was more preferred in normal tissues than in $\mathrm{HBV}(+) \mathrm{HCC}$ tissues. Other pre-miRNAs such as hsa-mir-23b showed a similar phenomenon. The above cases showed consistent patterns of expression ratios between all NT pairs, but diversities among NT pairs were observed in several pre-miRNAs, such as has-mir30c-2 (Figure 4d).

In summary, the $5 p$ and $3 p$ arm selection preferences are not always consistent between normal and tumor tissues, implying there is another selection mechanism, in addition to the hydrogen-bonding-based selection rule. If so, this selection mechanism may play a regulatory role in the oncogenesis pathway of HCC.

\section{Arm selection preference between normal and tumor tissues}

In our previous study, we showed that the arm selection preference of the same orthologous pre-miRNAs can vary between species, so that some species preferred the $5 p$ arm and the other preferred the 3p arm [19]. In Figure 4a, the expression ratio bars of the same pre-miRNAs showed different directions (upward or downward) between libraries. An upward bar means that the $5 \mathrm{p}$ arm was the major arm, while a downward bar means that the $3 p$ arm was the major arm. Therefore, we were curious about whether different arm selection preferences can be found between tissues, especially between the normal and HBV (+)HCC libraries.

We first examined whether the arm selection preference of pre-miRNA annotated by miRBase is consistent with the NGS expression data from the three normal and two $\mathrm{HBV}(+) \mathrm{HCC}$ libraries. Table 3 shows that the arm selection preferences of 13 pre-miRNAs were opposite to the miRBase annotation in more than half of the libraries studied. According to the miRBase annotation, hsa-mir-5111, hsa-mir-382, hsa-mir-548h-3 encode mature miRNA at only their $5 \mathrm{p}$ arm. In more than half of the analyzed libraries, however, we not only detected mature miRNAs at their 3p arms but also observed that the newly detected $3 p$ arms had a higher expression level than the originally annotated 5p arms. In addition, the newly detected 5p arm of hsa-mir-1307 also had a higher expression level than the originally annotated $3 \mathrm{p}$ arm.

For the pre-miRNAs originally annotated to encode miRNAs at both arms, the major arms of hsa-mir-30c-2, hsa-mir-30b, hsa-mir-106b and hsa-mir-500a were their $5 \mathrm{p}$ arms, while the major arms of hsa-mir-126, hsa-mir377, hsa-mir-193b, hsa-mir-505 and hsa-mir-664 were their 3p arms. From the NGS expression data, however, we found that their major arms and minor arms were switched, contrary to the miRBase annotation. Among them, hsa-mir-500a, hsa-mir-548h-3, hsa-mir-664 and hsa-mir-1307 were the extreme cases, at which the major-minor switch was observed in all of the analyzed libraries, including three normal and two $\mathrm{HBV}(+) \mathrm{HCC}$ libraries. In conclusion, the major and minor arms of pre-miRNA can vary among tissues.

Since the arm selection preference annotated by miRBase was inconsistent with our NGS expression data, we

Table 3 The pre-miRNAs whose arm selection preferences are not consistent with the miRBase annotation.

\begin{tabular}{lllllll}
\hline pre-miRNA & Location & N1 & N2 & N3 & T1 & T2 \\
\hline hsa-mir-30c-2 & MA:7-29;mi:47-68 & - & $Y$ & $Y$ & $Y$ & - \\
hsa-mir-30b & MA:17-38;mi:55-76 & $Y$ & $Y$ & $Y$ & - & - \\
hsa-mir-126 & mi:15-35;MA:52-73 & $Y$ & - & $Y$ & $Y$ & - \\
hsa-mir-106b & MA:12-32;mi:52-73 & $Y$ & - & $Y$ & $Y$ & - \\
hsa-mir-377 & mi:7-28;MA:45-66 & $Y$ & $Y$ & $Y$ & $Y$ & - \\
hsa-mir-382 & 5p:11-32 & $Y$ & $Y$ & $Y$ & $Y$ & - \\
hsa-mir-511-1 & 5p:16-36 & $Y$ & $Y$ & $Y$ & - & - \\
hsa-mir-193b & mi:14-35;MA:51-72 & $Y$ & $Y$ & $Y$ & - & - \\
hsa-mir-500a & MA:13-35;mi:52-73 & $Y$ & $Y$ & $Y$ & $Y$ & $Y$ \\
hsa-mir-505 & mi:15-36:MA:51-72 & $Y$ & $Y$ & $Y$ & - & $Y$ \\
hsa-mir-548h-3 & 5p:29-50 & $Y$ & $Y$ & $Y$ & $Y$ & $Y$ \\
hsa-mir-664 & mi:11-34;MA:49-71 & $Y$ & $Y$ & $Y$ & $Y$ & $Y$ \\
hsa-mir-1307 & 3p:80-101 & $Y$ & $Y$ & $Y$ & $Y$ & $Y$ \\
\hline
\end{tabular}

' $\mathrm{Y}$ ' indicates that the major-to-minor arm switch event was observed in the corresponding library; while '-' means "not observed". N1, N2, N3, T1 and T2 denote the SRX018957, SRX018958, SRX018959, SRX018965 and SRX018967 libraries, respectively. 
investigated whether arm selection preference could vary between tissues. In other words, we investigated whether the $5 p$ arm and $3 p$ arm miRNA derived from the same pre-miRNA have reversed tissue expression preferences without considering the miRBase annotation. For each pre-miRNA, we first tabulated the expression ratios of $5 p$ arm to $3 p$ arm miRNAs in three normal and two HBV $(+)$ HCC libraries (Additional File 3), excluding the 743 premiRNAs rarely expressed throughout all examined samples (the sum of total reads count in all samples $<100$ ). As a consequence, each pre-miRNA had five values. Then, we conducted the generalized linear model test to detect the differential arm ratios between normal and tumor with $\log 2$ transform as the link function. We indeed found several statistically significant pre-miRNAs, among which hsa-mir-30b and hsa-mir-193b are the perfect cases (Figure 5). In hsa-mir-30b, the arm rations are significantly tissue dependent. Their $3 p$ arm miRNAs had higher expression levels in the normal tissues, while their $5 \mathrm{p}$ arm miRNAs were predominated in the $\mathrm{HBV}(+) \mathrm{HCC}$ libraries ( $\mathrm{p}$ value $=0.013$ ). In contrast, the $5 \mathrm{p}$ arm of hsamir-193b was the major miRNA in normal libraries and the $3 p$ arm miRNA was the major one in $\mathrm{HBV}(+) \mathrm{HCC}$ libraries ( $\mathrm{p}$ value $=0.007$ ).

In this study, we show that although the $5 \mathrm{p}$ arm and $3 p$ arm miRNAs are derived from the same gene locus and transcribed by the same transcription factors, they have significant reversal expression preference between two tissues. We therefore conclude that there was another selection mechanism, in addition to the hydrogen-bonding-based selection rule. However, any factors affecting the precise measurement of miRNA's expression levels, such as PCR artifacts and unequal degradation rates, could also be possible explanations of the reversal tissue preference. During the sample preparation procedure of NGS, RNA molecules were amplified with PCR after adapter ligation. If the $5 p$ arm and $3 p$ arm miRNAs have different affinities with adapters, they may have unequal percentages of amplification, causing biased estimates of expression levels and false reversal tissue preference.

After miRNA/miRNA* (in terms of abundance) duplex is unwound, the miRNA is incorporated into RISC and usually has longer life, while the miRNA* is degraded soon. In addition to the protection of RISC, the nucleotide composition of the RNA molecule may also have unequal resistances to RNase. Therefore, the detected read counts by NGS may not really reflect the true miRNA's expression levels, causing biased expression preferences.

Although we can not completely rule out other factors causing biased measurement of miRNA's expression level, we presented a statistically significant result of miRNA's reversal preference in normal and tumor tissues. This new observation deserves further investigation.

\section{Materials and methods}

\section{Collecting and processing of sequence reads}

We first downloaded sequence reads with the accession number SRP002272 from NCBI SRA. This accession contains 15 libraries from different liver tissues. All library information is given in Table 1 . All of the 15 libraries were sequenced using the Illumina (Solexa) small RNA sequencing technology. After downloading, all sequence reads were first processed to remove the 3' end adapter and the ones with adapter detected and trimmed were called "clean reads". In view of the length distribution of mature miRNAs, only 18 25 nt clean reads were collected for analysis. Moreover, for higher confidence, only the unique clean reads with read count $\geqq 2$ were mapped back to human pre-miRNAs (miRBase 16).

\section{The criteria of mapping sequence reads back to pre- miRNAs}

In previous studies using NGS reads to quantify miRNA expression levels, nucleotide variations were usually allowed when mapping sequence reads back to the genomes or the reference miRNAs [28], resulting in ambiguously mapped loci caused by the high similarity between human mature miRNAs, such as hsa-miR-548a, hsa-miR-548b and hsa-miR-548z. In order to eliminate this type of ambiguity, we mapped the sequence reads back to pre-miRNAs with bowtie [30] by allowing no mismatch, as suggested previously $[11,19]$.

In this study, we map the sequence reads to pre-miRNAs rather than the mature miRNAs. We then have additional constraint to avoid random match. Mature miRNAs have many variants with different length, named isomiR. The isomiRs shift from their corresponding miRBase reference miRNAs in terms of location. When sequence reads were mapped back to mature miRNAs, the alignment shift may result in mismatches. Therefore, in addition to the perfect match constraint, we adopted an alternative procedure. In order to exclude random match, the difference in start position between mature miRNA and mapped reads must be equal to or less than two nucleotides and the difference in the end position between mature miRNA and mapped reads must be equal to or less than five nucleotides.

Previous studies reported nucleotide additions at the 3' end of miRNAs [10-12,26,27], which may cause mismatches. Therefore, following Fernandez-Valverde's strategy [31], we trimmed the last 3' end mismatch one by one until the perfect-match reads are at least 18 nucleotides in length.

\section{Analyzing the classes of sequence reads}

The non-miRNA sequence reads were further classified into nine classes by mapping to different data sets with bowtie [30] by allowing one-nucleotide variation. The 

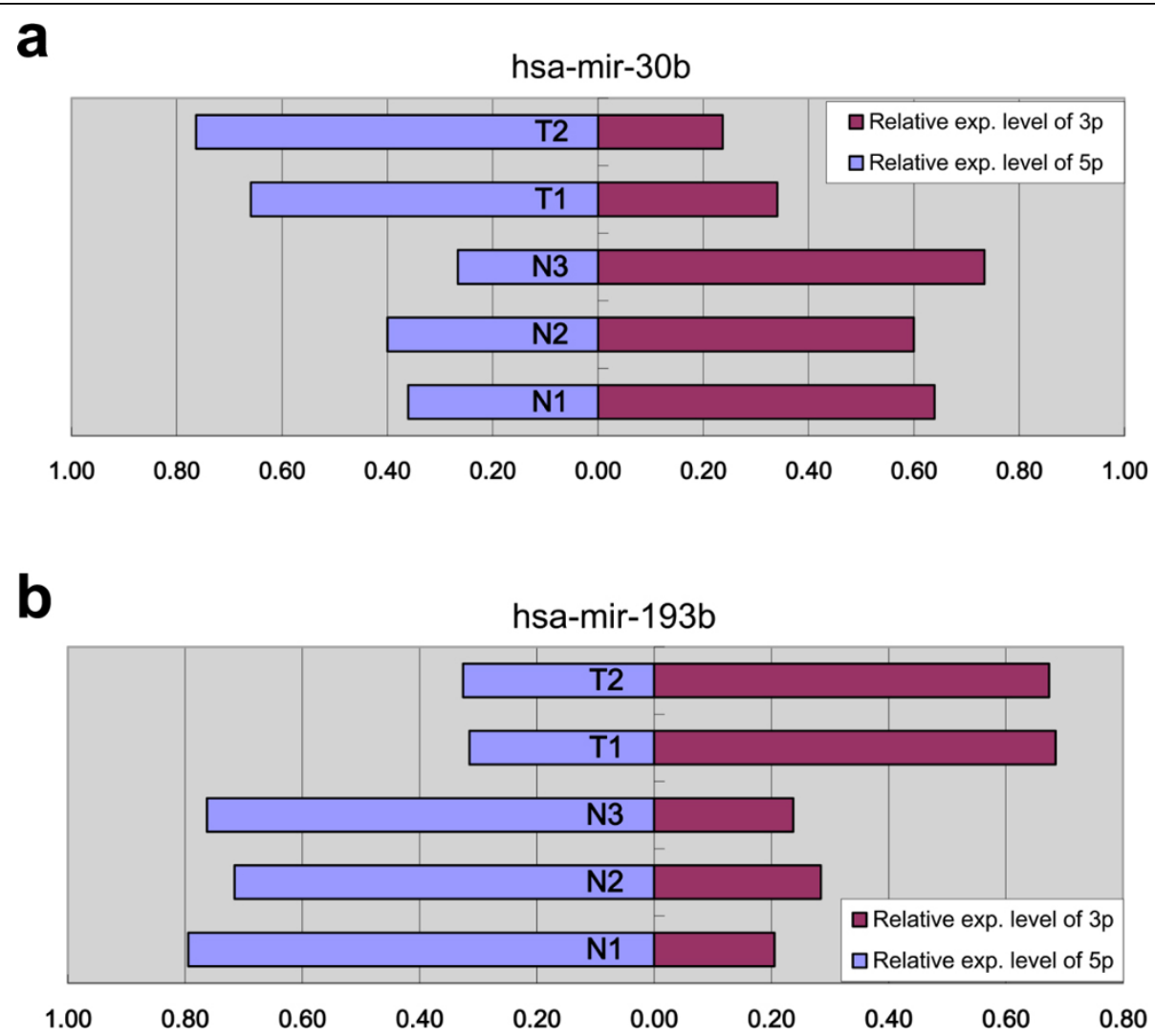

Figure 5 Arm selection preferences of $5 p$ arm and $3 p$ arm differ between normal liver and HBV(+)HCC tissues. In hsa-mir- $30 b$, the $5 p$ arm miRNA is preferred in tumor tissue, whereas the $3 p$ arm miRNA is preferred in normal tissue ( $p$ value $=0.013$ ). In hsa-mir-193b, the 5p arm miRNA is preferred in normal tissue, whereas the $3 p$ arm miRNA is preferred in tumor tissue ( $p$ value $=0.007$ ).

sequences of mRNAs came from the records with NM or XM accession prefix in RefSeq 47 [32]. The sequences of tRNAs were downloaded from the Genomic tRNA database [33]. The sequences of rRNAs were provided by the silva database [34]. The sequences of snoRNAs, scaRNAs and snRNAs were downloaded from NONCODE [35]. The sequences of other ncRNAs came from the records with NR or XR accession prefix in RefSeq 47 [32]. The sequence reads not belonging to any RNA classes were uploaded to RepaetMasker for identifying repeat elements [36]. The sequence reads not belonging to any of the previous classes were classified into the unknown class.

\section{Examining Drosha and Dicer expression levels in liver tissues}

Total RNA was isolated from the frozen tissues using a guanidium isothiocyanate/CsCl method. RNA was quantified by spectrophotometry at $260 \mathrm{~nm}$. Complementary DNA (cDNA) was prepared from the 2 microgram total RNA of paired HCCs and nontumorous liver samples. One microliter reverse transcription product, 1.25 units Pro Taq polymerase (Protech Technology Enterprise,
Taipei, Taiwan), Pro Taq buffer, and $200 \mu \mathrm{M}$ dATP, dCTP, dGTP, and dTTP (each) were mixed with primer pairs for Dicer, Drosha, PBGD and S26 in a total volume of $30 \mu \mathrm{l}$. PCR was performed in an automated DNA thermal cycler, 30 cycles for Dicer and Drosha, 28 cycles for PBDG and S26, with initial heating at $94{ }^{\circ} \mathrm{C}$ for 2 minutes, followed by the Touchdown PCR: 30 or 28 cycles of $94{ }^{\circ} \mathrm{C}$ for 30 seconds, annealing for 1 minute (the annealing temperature is reduced by $1{ }^{\circ} \mathrm{C}$ per 2 cycles from $65^{\circ} \mathrm{C}$ to $55^{\circ}$ $\mathrm{C}$ for 20 cycles, than constant the annealing temperature at $55^{\circ} \mathrm{C}$ for final 10 or 8 cycles), $72{ }^{\circ} \mathrm{C}$ for 1 minute, and final $72{ }^{\circ} \mathrm{C}$ for 10 minutes. Primers for amplified genes were as follows: Dicer-F (5'- GTACGACTACCACAAGTACTTC -3'), Dicer-R (5' - ATAGTACACCTGCCAGACTGT -3'), Drosha-F (5'-GTGCTGTCCATGCAC CAGATT -3'), Drosha-R(5'-TGCATAACTCAACTGTGCAG G -3'), S26-F (5'-CCGTGCCTCCAAGATGACAAAG-3') and S26-R (5'-TGTCTGGTAACGGCAA TGCGGCT-3').

\section{Funding}

This work was supported by grants from National Science Council of Taiwan (NSC99-2628-B-001-009-MY3). 


\section{Additional material}

Additional File 1: RNA classes of the sequence reads in libraries. We first mapped the sequence reads back to pre-miRNAs, followed by mapping the non-miRNA reads back to different datasets for identifying their RNA categories

Additional File 2: Expression of Dorsha and Dicer in HCV-positive and HCV-negative samples. Reverse transcription-polymerase chain reaction (RT-PCR) was used to determine the mRNA levels of Dicer and Drosha in HCCs with hepatitis C virus ( $\mathrm{HCV}$ ) infection or not and their non-tumor liver samples. ribosomal protein $\$ 26$ was used as internal control.

Additional File 3: The mapping result of all miRNA reads in all libraries

\section{Acknowledgements}

We thank NCBI SRA for organizing and releasing the sequence reads of liver samples.

This article has been published as part of BMC Systems Biology Volume 6 Supplement 2, 2012: Proceedings of the 23rd International Conference on Genome Informatics (GIW 2012). The full contents of the supplement are available online at http://www.biomedcentral.com/bmcsystbiol/supplements/ 6/S2.

\section{Author details}

${ }^{1}$ Genomics Research Center, Academia Sinica, Taipei, Taiwan. ${ }^{2}$ Department of Medical Education and Research, Kaohsiung Veterans General Hospital, Kaohsiung, Taiwan. ${ }^{3}$ Graduate Institute of Pathology, National Taiwan University, Taipei, Taiwan. ${ }^{4}$ Biodiversity Research Center, Academia Sinica, Taipei, Taiwan. ${ }^{5}$ Department of Ecology and Evolution, University of Chicago, Chicago, IL 60637, USA.

\section{Authors' contributions}

SCL conducted this analysis and wrote the draft of the manuscript. KWT, HWP and YMJ were responsible for tissues collection and performed the PCR experiments. MRH dealt with the conducted the generalized linear model test. WHL supervised this project and edited the manuscript.

\section{Competing interests}

The authors declare that they have no competing interests.

\section{Published: 12 December 2012}

\section{References}

1. Wang $X C$, Tian $L L, W u H L$, Jiang $X Y$, Du LQ, Zhang $H$, Wang $Y Y, W u H Y$, Li DG, She $Y$, et al: Expression of miRNA-130a in nonsmall cell lung cancer. Am J Med Sci 2010, 340(5):385-388.

2. Cohn DE, Fabbri M, Valeri N, Alder H, Ivanov I, Liu CG, Croce CM, Resnick KE: Comprehensive miRNA profiling of surgically staged endometrial cancer. Am J Obstet Gynecol 2010, 202(6):656.e1-8.

3. Chan SH, Wu CW, Li AF, Chi CW, Lin WC: miR-21 microRNA expression in human gastric carcinomas and its clinical association. Anticancer Res 2008, 28(2A):907-911.

4. Tan Y, Zhang B, Wu T, Skogerbo G, Zhu X, Guo X, He S, Chen R: Transcriptional inhibiton of Hoxd4 expression by miRNA-10a in human breast cancer cells. BMC Mol Biol 2009, 10:12.

5. Hausler SF, Keller A, Chandran PA, Ziegler K, Zipp K, Heuer S, Krockenberger $M$, Engel JB, Honig A, Scheffler $M$, et al: Whole bloodderived miRNA profiles as potential new tools for ovarian cancer screening. Br J Cancer 2010, 103(5):693-700.

6. Hao Y, Zhao Y, Zhao X, He C, Pang X, Wu TC, Califano JA, Gu X: Improvement of prostate cancer detection by integrating the PSA test with miRNA expression profiling. Cancer Invest 2011, 29(4):318-324.

7. Mezzanzanica D, Canevari S, Cecco LD, Bagnoli M: miRNA control of apoptotic programs: focus on ovarian cancer. Expert Rev Mol Diagn 2011, 11(3):277-286.
8. Li SC, Liao YL, Ho MR, Tsai KW, Lai CH, Lin WC: miRNA arm selection and isomiR distribution in gastric cancer. BMC Genomics 2012, 13(Suppl 1):S13.

9. Hou J, Lin L, Zhou W, Wang Z, Ding G, Dong Q, Qin L, Wu X, Zheng Y, Yang $Y$, et al: Identification of miRNomes in human liver and hepatocellular carcinoma reveals miR-199a/b-3p as therapeutic target for hepatocellular carcinoma. Cancer Cell 2011, 19(2):232-243.

10. Landgraf P, Rusu M, Sheridan R, Sewer A, lovino N, Aravin A, Pfeffer S, Rice A, Kamphorst $\mathrm{AO}$, Landthaler $\mathrm{M}$, et al: A mammalian microRNA expression atlas based on small RNA library sequencing. Cell 2007, 129(7):1401-1414.

11. Morin RD, O'Connor MD, Griffith M, Kuchenbauer F, Delaney A, Prabhu AL, Zhao $Y$, McDonald $H$, Zeng $T$, Hirst $M$, et al: Application of massively parallel sequencing to microRNA profiling and discovery in human embryonic stem cells. Genome Res 2008, 18(4):610-621.

12. Ebhardt HA, Tsang HH, Dai DC, Liu Y, Bostan B, Fahlman RP: Meta-analysis of small RNA-sequencing errors reveals ubiquitous post-transcriptional RNA modifications. Nucleic Acids Res 2009, 37(8):2461-2470.

13. Suzuki HI, Miyazono K: Emerging complexity of microRNA generation cascades. J Biochem 2011, 149(1):15-25.

14. Trabucchi M, Briata P, Filipowicz W, Rosenfeld MG, Ramos A, Gherzi R: How to control miRNA maturation? RNA Biol 2009, 6(5):536-540.

15. Tsai KW, Wu CW, Hu LY, Li SC, Liao YL, Lai CH, Kao HW, Fang WL, Huang $\mathrm{KH}$, Chan WC, et al: Epigenetic regulation of miR-34b and miR-129 expression in gastric cancer. Int J Cancer 2011, 129:2600-2610.

16. Griffiths-Jones S, Grocock RJ, van Dongen S, Bateman A, Enright AJ: miRBase: microRNA sequences, targets and gene nomenclature. Nucleic Acids Res 2006, 34(Database issue):D140-144.

17. Bartel DP: MicroRNAs: genomics, biogenesis, mechanism, and function. Cell 2004, 116(2):281-297

18. Schwarz DS, Hutvagner G, Du T, Xu Z, Aronin N, Zamore PD: Asymmetry in the assembly of the RNAi enzyme complex. Cell 2003, 115(2):199-208.

19. Li SC, Chan WC, Ho MR, Tsai KW, Hu LY, Lai CH, Hsu CN, Hwang PP, Lin WC: Discovery and characterization of medaka miRNA genes by next generation sequencing platform. BMC Genomics 2010, 11(Suppl 4):S8.

20. Griffiths-Jones S, Hui JH, Marco A, Ronshaugen M: MicroRNA evolution by arm switching. EMBO Rep 2011, 12(2):172-177

21. Vaz C, Ahmad HM, Sharma P, Gupta R, Kumar L, Kulshreshtha R, Bhattacharya $A$ : Analysis of microRNA transcriptome by deep sequencing of small RNA libraries of peripheral blood. BMC Genomics 2010, 11:288.

22. Li SC, Chan WC, Lai CH, Tsai KW, Hsu CN, Jou YS, Chen HC, Chen CH, Lin WC: UMARS: Un-MAppable Reads Solution. BMC Bioinformatics 2011, 12(Suppl 1):S9.

23. Glazov EA, Cottee PA, Barris WC, Moore RJ, Dalrymple BP, Tizard ML: A microRNA catalog of the developing chicken embryo identified by a deep sequencing approach. Genome Res 2008, 18(6):957-964.

24. Roberts $A P$, Lewis $A P$, Jopling $C L$ : miR-122 activates hepatitis $C$ virus translation by a specialized mechanism requiring particular RNA components. Nucleic Acids Res 2011, 39:7716-7729.

25. Bihrer V, Friedrich-Rust M, Kronenberger B, Forestier N, Haupenthal J, Shi Y Peveling-Oberhag J, Radeke HH, Sarrazin C, Herrmann E, et al: Serum miR122 as a Biomarker of Necroinflammation in Patients With Chronic Hepatitis C Virus Infection. Am J Gastroenterol 2011, 106:1663-1669.

26. Siomi MC, Siomi H: Characterization of endogenous human Argonautes and their miRNA partners in RNA silencing. Nucleic Acids Symp Ser (Oxf) 2008, 52: 59-60.

27. Reid JG, Nagaraja AK, Lynn FC, Drabek RB, Muzny DM, Shaw CA, Weiss MK, Naghavi AO, Khan M, Zhu H, et al: Mouse let-7 miRNA populations exhibit RNA editing that is constrained in the 5 '-seed/ cleavage/anchor regions and stabilize predicted mmu-let-7a:mRNA duplexes. Genome Res 2008 18(10):1571-1581.

28. Wheeler BM, Heimberg AM, Moy VN, Sperling EA, Holstein TW, Heber S, Peterson KJ: The deep evolution of metazoan microRNAs. Evol Dev 2009, 11(1):50-68.

29. Li SC, Liao YL, Chan WC, Ho MR, Tsai KW, Hu LY, Lai CH, Hsu CN, Lin WC: Interrogation of rabbit miRNAs and their isomiRs. Genomics 2011, 98(6):453-459.

30. Langmead B, Trapnell C, Pop M, Salzberg SL: Ultrafast and memoryefficient alignment of short DNA sequences to the human genome. Genome Biol 2009, 10(3):R25.

31. Fernandez-Valverde SL, Taft RJ, Mattick JS: Dynamic isomiR regulation in Drosophila development. RNA 2010, 16(10):1881-1888. 
32. Pruitt KD, Tatusova T, Klimke W, Maglott DR: NCBI Reference Sequences: current status, policy and new initiatives. Nucleic Acids Res 2009, 37(Database issue):D32-36.

33. Chan PP, Lowe TM: GtRNAdb: a database of transfer RNA genes detected in genomic sequence. Nucleic Acids Res 2009, 37(Database issue):D93-97.

34. Pruesse E, Quast C, Knittel K, Fuchs BM, Ludwig W, Peplies J, Glockner FO: SILVA: a comprehensive online resource for quality checked and aligned ribosomal RNA sequence data compatible with ARB. Nucleic Acids Res 2007, 35(21):7188-7196.

35. Liu C, Bai B, Skogerbo G, Cai L, Deng W, Zhang Y, Bu D, Zhao Y, Chen R: NONCODE: an integrated knowledge database of non-coding RNAs. Nucleic Acids Res 2005, 33(Database issue):D112-115.

36. RepeatMasker:[http://www.repeatmasker.org/].

doi:10.1186/1752-0509-6-S2-S14

Cite this article as: Li et al: MicroRNA $3^{\prime}$ end nucleotide modification patterns and arm selection preference in liver tissues. BMC Systems Biology 2012 6(Suppl 2):S14

\section{Submit your next manuscript to BioMed Central} and take full advantage of:

- Convenient online submission

- Thorough peer review

- No space constraints or color figure charges

- Immediate publication on acceptance

- Inclusion in PubMed, CAS, Scopus and Google Scholar

- Research which is freely available for redistribution

Submit your manuscript at www.biomedcentral.com/submit 